\title{
Belief revision as a problem of scientific epistemology
}

\author{
Yaroslav V. Shramko ${ }^{1}$ \\ ${ }^{1}$ Kryvyi Rih State Pedagogical University, 54 Gagarin Ave., Kryvyi Rih, 50086, Ukraine
}

\begin{abstract}
The fundamental question that must be answered by any theory of knowledge that claims to be adequate is the question of how it is possible to change our knowledge. The very fact of change undoubtedly takes place, and the problem is to theoretically explicate this fact. The methodological significance of this issue is due to the fact that changing knowledge means nothing more than its development, namely, the question of the ways and means of developing our knowledge is of central importance both for the logic and methodology of science, and for general epistemology. This work is of a review character, and aims to draw the reader's attention to a new promising direction in the modern theory of knowledge called "belief revision".
\end{abstract}

Keywords: belief revision, scientific epistemology

Основополагающим вопросом, на который должна ответить всякая теория познания, претендующая на адекватность, является вопрос о том, как возможно и каким образом осуществляется изменение нашего знания. Сам факт изменения несомненно имеет место, и проблема заключается в том, чтобы теоретически эксплицировать данный факт. Методологическая значимость этого вопроса обусловлена тем обстоятельством, что изменение знания означает не что иное, как его развитие, а именно вопрос о путях и способах развития нашего знания имеет центральное значение как для логики и методологии науки, так для общей эпистемологии.

Настоящая работа носит обзорный характер и имеет целью привлечь внимание читателя к новому перспективному направлению в современной теории познания, за которым в англоязычной литературе закрепилось название "belief revision".

Прежде всего представляется уместным уточнить некоторые ключевые термины, которые использованы в заглавии. Под "ревизией" знания следует понимать его пересмотр. Очевидно, что время от времени в силу различных причин (например, в результате изменения нас самих или изменения окружающей нас действительности) мы подвергаем наши знания пересмотру с целью решить, какие из этих знаний устарели и должны быть отброшены, а какие нужно сохранить и, быть может, развить дальше. Таким образом, если мы хотим получить ответ на вопрос, как происходит изменение нашего знания, мы должны объяснить, каковы те правила и методы, по которым осуществляется его пересмотр. Второе, еще более важное уточнение относится к самому термину “знание”. Очевидно, что ограничение сущностей образующих то, что может быть названо эпистемическим состоянием субъекта, только сферой

KMITO 1999: Conference on Computer Simulation and Information Technology in Education, April 19-21, 1999, Kryvyi Rih, Ukraine

Өshramko@rocketmail.com (Y.V. Shramko)

\# https://kdpu.edu.ua/shramko (Y. V. Shramko)

CC Copyright for this paper by its authors, published by Academy of Cognitive and Natural Sciences (ACNS)

作

which permits unrestricted use, distribution, and reproduction in any medium, provided the original work is properly cited.

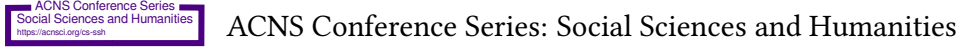


знания, означало бы неоправданное сужение самого предмета теории познания. При построении теоретической модели познавательной деятельности необходимо учитывать не только те ее результаты, которые обладают стопроцентной достоверностью и являются несомненно истинными (то есть, собственно знание), но также и все те положения, которые субъект только считает истинными. Совокупность таких положений может быть названо “мнением” субъекта (по тому или иному вопросу), или его “убеждениями”. По-видимому, правильным будет предположить, что знания субъекта составляет собственное подмножество множества его убеждений. Здесь важно отвлечься от той эмоциональной окрашенности, с которой обычно связано употребление слова “убеждение” в обыденном (русском) языке (в таких словосочетаниях как “идейные убеждения”, “стойкие убеждения” и т.п.). В контексте настоящей работы данный термин лишен какой бы то ни было этической или идеологической нагрузки и понимается исключительно в смысле "те положения, которые субъект в данный момент считает истинными”. Учитывая, однако, то обстоятельство, что в некоторых случаях бывает крайне трудно полностью отвлечься от нежелательных ассоциаций эмоциональноэтического характера, время от времени мы все-же будем употреблять термин “знание”, придавая ему тот расширительный смысл, о котором речь шла выше.

Итак, далее речь пойдет о том, каким образом мы пересматриваем наши убеждения. При этом мы не будем затрагивать вопрос, почему мы это делаем. Вопрос о причинах, которые иногда побуждают нас пересмотреть (подвергнуть ревизии) то, во что мы ранее верили, выходит за рамки теории познания и не является собственно эпистемологическим вопросом. Также второстепенное значение для чистой теории познания имеет вопрос о той “действительности”, к которой относятся наши знания. Вопрос о природе такого рода действительности равно как и вопрос о самом ее существовании представляет собой в сущности философски открытую проблему, которая вряд ли может быть решена окончательно. По крайней мере, она не может быть решена в рамках одной лишь теории познания. Поэтому при построении эпистемологических моделей целесообразно вообще не поднимать такого рода метафизические проблемы. Нас также не будут интересовать психолингвистические (или психофизические) процессы, происходящие в голове субъекта когда он меняет свое мнение, и обеспечивающие психологический механизм такого изменения. Эпистемология рассматривает знания и убеждения субъекта как некоторое объективированное знание, как мир объективного содержания мышления (“третий мир” Карла Поппера). Понимаемое таким образом знание представляет собой некоторую (и довольно сильную) эпистемологическую идеализацию необходимую для создания когнитивной модели познавательной деятельности. При таком понимании, вопрос о конкретном материальном носителе убеждений перестает быть существенным и наличие человеческого мозга вообще не является обязательным. Такого рода знание может быть смоделировано как результат деятельности “идеального субъекта”, например, как состояние компьютера или компьютерной программы.

Основными понятиями, образующими каркас рассматриваемой когнитивной модели, являются понятие “эпистемического состояния" и понятие “познавательной операции”. Первое из этих понятий служит для представления возможного состояния познающего субъекта в некоторый момент времени. Мы предполагаем, что такого рода состояние 
является заданным, если нам известны все те положения, которые индивид принимает в данный момент времени, то есть, в истинности которых он убежден. Таким образом, эпистемическое состояние субъекта есть ни что иное как множество его убеждений. С логической точки зрения это множество может быть описано как некоторое множество высказываний (а именно, множество всех тех высказываний, относительно которых субъект верит, что они являются истинными). Важно отметить, что эпистемология имеет дело с рациональным субъектом, то есть, субъектом, познавательная деятельность которого организована рациональным образом. В этой связи возникает вопрос о том, когда субъект может считаться рациональным, а это есть вопрос о критериях рациональности, которым должны подчиняться эпистемические состояния субъекта. Обычно принимаются следующие два требования рациональности:

(1) Множество убеждений субъекта должно быть непротиворечивым.

(2) Субъект обязан принимать все логические следствия принимаемых им убеждений.

Убеждения, удовлетворяющие данным требованиям, считаются рациональными. Эти требования являются очевидно довольно сильными идеализациями. Так, например, в действительности убеждения субъекта иногда (а возможно и часто) могут противоречить друг другу. Мы однако считаем, что противоречивые убеждения не представляют особого теоретического интереса, поскольку не совсем ясно, каким образом такого рода убеждения могут быть подвергнуты рациональному анализу. Поэтому, если вдруг обнаруживается, что множество убеждений индивида является противоречивым, то такое положение дел считается ненормальным и рациональный индивид, в соответствии с требованием непротиворечивости, обязан предпринять все необходимые действия для устранения противоречия, или, по крайней мере, для его изоляции. Что касается второго требования, то его не следует понимать в том смысле, что субъект действительно осознает все логические следствия своих убеждений. Скорее, это требование отражает эпистемические обязательства рационального субъекта. Так, например, если индивид верит, что все люди смертны, а также верит, что Сократ человек, то тогда он обязан принять утверждение, что Сократ смертен, даже если он явным образом никогда не задумывался над этим последним вопросом. Если же этот индивид, вопреки своим первым двум убеждениям, будет отказываться принять истинность последнего утверждения, то такой индивид будет признан нами нерациональным (или иррациональным), что, очевидно, является вполне обоснованным.

Второе требование иногда формулируется еще и следующим образом:

(2’) Множество убеждений субъекта должно быть замкнуто по отношению логического следования.

Формально это может быть представлено с помощью особой операции замыкания $C n$. Пусть Х есть некоторое множество высказываний. Тогда $C n(\mathrm{X})$ есть множество всех логических следствий из $\mathrm{X}$, которое называется замыканием $\mathrm{X}$. $C n$ должна удовлетворять следующим стандартным условиям:

(a) $\mathrm{X} \subseteq C n(\mathrm{X})$; 
(b) Если $\mathrm{X} \subseteq \mathrm{Y}$, то $C n(\mathrm{X}) \subseteq C n(\mathrm{Y})$;

(c) $C n(\mathrm{X})=C n(C n(\mathrm{X}))$.

Используя операцию замыкания, мы вводим следующее понятие "системы убеждений”:

\section{Определение 1.}

$\mathrm{X}$ есть (неабсурдная) система убеждений если и только если $\mathrm{X}=C n(\mathrm{X})$.

Следующее важное понятие - это понятие “познавательной операции" или “познавательного действия". Именно это понятие дает нам возможность отразить основные типы изменения наших систем убеждений. Пусть К есть некоторая система убеждений. Тогда относительно К возможны следующие познавательные операции, которые приводят к изменению K:

1. Расширение. Эта операция применяется, когда мы хотим расширить наши убеждения за счет добавления новых убеждений к уже имеющимся. При этом мы надеемся, что получающаяся в результате новая система убеждений является непротиворечивой, хотя одна лишь операция расширения знаний сама по себе, конечно, не может этого гарантировать. Обозначим операцию расширения знаний посредством “+”. То есть, если $\mathrm{K}$ - имеющаяся система убеждений, а $A$ - некоторое высказывание, то $\mathrm{K}+A$ есть результат расширения К посредством высказывания $A$.

2. Сокращение. Эта операция применяется, когда мы считаем нужным отказаться от некоторого убеждения, то есть, когда мы удаляем это убеждение из нашей системы убеждений. Эта операция обозначается посредством “ $\div$ ”: $\mathrm{K} \div A$ есть результат сокращения системы убеждений $\mathrm{K}$ за счет высказывания $A$.

3. Ревизия. Эта операция применяется, если мы пришли к необходимости признать истинность некоторого высказывания, которое является несовместимым с нашей прежней системой убеждений. В этом случае мы добавляем данное высказывание к нашей системе убеждений, и одновременно осуществляем пересмотр (ревизию) наших старых убеждений с целью сделать их совместимыми с вновь принятым высказыванием. Если операцию ревизии обозначить посредством “*”, то тогда $\mathrm{K} * A$ будет результатом ревизии системы убеждений $\mathrm{K}$ относительно высказывания $A$.

Ни одна из этих познавательных операций не сводится к простому механическому одноразовому действию. Так, например, если мы расширяем имеющуюся систему убеждений за счет некоторого высказывания, недостаточно просто добавить это высказывание к множеству старых убеждений. Ведь то, что получится в результате, также должно быть системой убеждений, то есть по определению 1 она должна быть замкнута по отношению логического следования. Иными словами, при добавлении нового убеждения к уже имеющимся, мы должны добавить сюда также и все логические следствия, которые отсюда вытекают. С другой стороны, если мы осуществляем сокращение наших знаний, недостаточно просто удалить некоторое высказывание из нашей системы убеждений. Дело в том, что мы должны также исключить и все те высказывания, из которых удаляемое высказывание логически следует, поскольку если этого не сделать, то удаляемое высказывание фактически вовсе не будет удалено, 
а неявным образом сохранится в системе убеждений. Далее, если два различных высказывания совместно влекут удаляемое убеждение, то одно из этих высказываний также должно быть удалено, и здесь мы оказываемся в ситуации выбора, который далеко не всегда является тривиальным.

Очевидно, что расширение и сокращение знания представляют собой в значительной степени идеальные познавательные действия, которые в чистом виде встречаются довольно редко. По-видимому наиболее типичной эпистемической операцией является ревизия, и процесс развития наших убеждений чаще всего происходит именно путем их пересмотра. В этой связи возникает интересный теоретический вопрос - является ли ревизия независимой познавательной операцией и нельзя ли попробовать свести ее к двум другим, то есть определить ревизию через расширение и сокращение. Оказывается, что такое сведение вполне возможно. По существу, операция ревизии представляет собой некоторое комплексное действие, заключающееся в том, что мы должны (1) включить некоторое новое высказывание $A$ в нашу систему убеждений и (2) принять все необходимые меры к тому, чтобы наша новая система убеждений была непротиворечивой. Первое из этих действий достигается путем расширения имеющейся системы убеждений за счет $A$, в то время как вторая цель может быть достигнута посредством предварительного удаления $\neg A$ (отрицание $A$ ) из нашей системы убеждений (сокращение). Иными словами, операция ревизии может быть эксплицирована как результат последовательного осуществления двух подопераций: (1) сокращение посредством $\neg A$ и (2) расширение за счет $A$. Таким образом, приходим к следующему определению, известному в литературе как “равенство Леви”:

\section{Определение 2 .}

$$
\mathrm{K} * A=(\mathrm{K} \div \neg A)+A \text {. }
$$

Это определение имеет очень большое эвристическое значение, поскольку с его принятием проблема теоретической экспликации процесса изменения наших знаний сводится к рассмотрению двух сравнительно простых познавательных операций расширению и сокращению знаний. Рассмотрим первую из этих операций. Очевидно, что расширение можно довольно легко определить, используя аппарат теории множеств. А именно, если мы хотим расширить нашу систему убеждений $\mathrm{K}$ за счет высказывания $A$, мы должны “механически” добавить это высказывание к $\mathrm{K}$ (осуществить теоретико-множественное объединение), а затем замкнуть получившееся множество высказываний $\mathrm{K}$ посредством операции замыкания $C n$ :

\section{Определение 3.}

$$
\mathrm{K}+A=C n(\mathrm{~K} \cup\{A\})
$$

Посредством данного определения операция расширения знаний вводится однозначным образом, не оставляя пространства для различных ее истолкований. А это значит, что вся проблема ревизии знаний фактически эквивалентна проблеме определения операции сокращения. Как ни парадоксально это звучит, но если мы хотим получить ответ на вопрос о том, каким образом осуществляется изменение (а 
значит и развитие) нашего знания, мы должны ответить на вопрос, как происходит его сокращение. Принимая же оптимистическую точку зрения, в соответствии с которой в процессе развития знания происходит его рост, мы приходим к следующему кардинальному выводу: проблема роста знания сводима к проблеме его сокращения. И если бы нам удалось найти для этой операции такое же четкое определение, как определение 3, то тогда проблема теоретической экспликации развития знания была бы решена однозначным образом.

К сожалению (а может быть и к счастью) однозначно определить операцию сокращения не удается. Основной причиной этого является отмеченная выше возможность “альтернативных ходов”, неизбежное появление при осуществлении сокращения ситуации неопределенности, когда мы оказываемся перед выбором, какое из нескольких высказываний удалить из системы наших убеждений, а какое оставить, и при этом не существует никаких чисто логических предпочтений в пользу того или иного высказывания.

Остановимся кратко на некоторых возможных подходах к определению операции сокращения. Пусть $\mathrm{X}$ есть некоторое множество высказываний и $A$ - некоторое высказывание. Определим множество $\mathrm{X} \perp A$ (читается “ $\mathrm{X}$ без $A$ ”) как множество всех максимальных подмножеств $\mathrm{X}$, таких что они не влекут $A$. Формально:

\section{Определение 4.}

$$
\begin{aligned}
& \text { (1) } \mathrm{y} \subseteq \mathrm{X} \\
& \mathrm{y} \in \mathrm{X} \perp A \Longleftrightarrow \quad(2) A \notin C n(\mathrm{y}) \\
& \text { (3) не существует множества У’ такого, что: У } \subset \text { У’ } \subseteq \mathrm{X} \text { и } A \notin C n\left(\mathrm{y}^{\prime}\right)
\end{aligned}
$$

Теперь можно было бы попробовать определить результат сокращения некоторой системы убеждений К посредством высказывания $A$ как пересечение всех элементов принадлежащих множеству $\mathrm{K} \perp A$ :

\section{Определение 5 .}

$$
\mathrm{K} \div A=\cap(\mathrm{K} \perp A)
$$

Определенная таким образом операция сокращения получила в литературе название “сокращение полного пересечения". Нетрудно видеть, что такого рода операция является излишне “перестраховочной”, она требует удалять из наших убеждений слишком многое, даже если мы этого не хотим. Например, если мы стоим перед выбором - отказаться от одного из каких-либо двух высказываний, то сокращение полного пересечения требует от нас удалить оба эти высказывания, что далеко не всегда представляется оправданным. Более того, легко может быть доказана следующая лемма:

Лемма 1. (Алчуррон и Макинсон)

Если “ $\div$ ” есть сокращение полного пересечения и $A \in \mathrm{K}$, то имеем: $B \in \mathrm{K} \div A \Longleftrightarrow B \in \mathrm{K}$ и $B \in C n(\neg A)$

\section{Доказательство:}


Доказательство предоставляется любознательному читателю в качестве упражнения.

Иными словами, результатом сокращения наших убеждений в соответствии с определением 5 будет лишь множество тех убеждений, которые логически следуют из одного только высказывания $\neg A$ ! Ясно, что такое сокращение не может быть признано удачным.

Другое возможное предложение заключается в том, чтобы выбрать из множества $\mathrm{K} \perp A$ один элемент и рассмотреть его как результат применения операции сокращения. Это значит, что мы вводим на множестве $\mathrm{K} \perp A$ некоторую функцию выбора, скажем $\delta$, которая выбирает из этого множества ровно один элемент $-\delta(\mathrm{K} \perp A)$ для каждого $A$. Тогда имеем следующее определение, посредством которого вводится так называемое "сокращение максимального выбора":

\section{Определение 6.}

$\mathrm{K} \div A=\delta(\mathrm{K} \perp A)$

Сокращение максимального выбора также имеет существенный недостаток - оно не оставляет возможности действовать достаточно осторожно. Так, если мы находимся перед выбором - удалить либо высказывание $A$, либо - высказывание $B$ и при этом не имеем абсолютно никаких резонов предпочесть одно из этих высказываний, может оказаться полезным отбросить оба эти высказывания, чтобы быть полностью уверенным в наших убеждениях. Например, пусть мы полагали, что госпожа Иванова имеет ровно два ребенка - мальчика и девочку, а затем узнали, что на самом деле ребенок у Ивановой только один, при этом о поле ребенка ничего не было сказано. Естественно, мы не можем сохранить оба имевшиеся ранее у нас убеждения "Иванова имеет мальчика" и “Иванова имеет девочку”. И хотя “объективно” одно из этих высказываний является истинным, но, поскольку мы не получили достаточно точной информации, будет разумным отбросить (по крайней мере пока, до получения необходимых уточняющих данных) оба эти убеждения и признать, что мы не уверены ни в том, что госпожа Иванова имеет мальчика, ни в том, что она имеет девочку. Такого рода стратегия к сожалению оказывается невозможной в рамках определения 6.

Более разумным представляется следующий путь. Мы вводим на множестве $\mathrm{K} \perp A$ некоторую функцию предпочтения, скажем $\gamma$, которая отбирает те элементы этого множества, которые являются более “предпочтительными”, более “достойны сохранения”, по сравнению с остальными множествами убеждений. Результатом сокращения будет тогда пересечение всех элементов из $\gamma(\mathrm{K} \perp A)$. Это есть "сокращение частичного пересечения".

\section{Определение 7.}

Если $\mathrm{K} \perp A$ непусто, то $\gamma(\mathrm{K} \perp A) \subseteq \mathrm{K} \perp A$ и $\mathrm{K} \perp A$ также непусто;

Если $\mathrm{K} \perp A$ пусто, то $\gamma(\mathrm{K} \perp A)=\{\mathrm{K}\}$.

\section{Определение 8.}




$$
\mathrm{K} \div A=\cap \gamma(\mathrm{K} \perp A)
$$

Нетрудно видеть, что сокращения полного пересечения и максимального выбора суть частные случаи сокращения частичного пересечения. Оказывается также, что свойства данной операции могут быть охарактеризованы посредством некоторого набора постулатов, которые должны для нее выполняться. Иными словами, операция частичного сокращения допускает построение определенной аксиоматической теории. Опишем кратко эти постулаты:

1. "Постулат замыкания" (closure): если $\mathrm{K}$ является системой убеждений, то $\mathrm{K} \div A$ также есть система убеждений.

(Иными словами, $\mathrm{K} \div A$ должно быть замкнуто по отношению логического следования, если таковым является само К.)

2. "Постулат успеха" (success): если $A \notin C n(\varnothing)$, то $A \notin \mathrm{K} \div A$.

(Успех сокращения очевидно заключается в том, что удаляемое высказывание не должно принадлежать результирующей системе убеждений. Однако, сокращение не может быть успешным, если мы попытаемся удалить из наших убеждений логически истинное высказывание (то есть закон логики). Тот факт, что высказывание $A$ является логической теоремой можно обозначить посредством $A \notin C n(\varnothing)$, поэтому постулат успеха имеет в качестве условия требование, что $A$ не является теоремой логики.)

3. "Постулат включения" (inclusion): $\mathrm{K} \div A \subseteq \mathrm{K}$.

(Получившаяся в результате сокращения система убеждений должна составлять подмножество исходной системы убеждений.)

4. "Постулат nустоть" (vacuity): если $A \notin \mathrm{K}$, то $\mathrm{K} \div A=\mathrm{K}$.

(Если мы попытаемся “удалить” из нашей системы убеждений высказывание, которое в действительности вовсе не принадлежит этой системе, то наша система убеждений просто останется без изменения - никакого сокращения не произойдет.)

5. "Постулат восстановления" (recovery): $\mathrm{K} \subseteq(\mathrm{K} \div A)+A$.

(В соответствии с этим постулатом, все наши знания должны быть восстановлены, если мы вначале сократим систему убеждений посредством высказывания $A$, а затем возвратим $A$ в нашу систему убеждений.)

6. "Постулат экстенсиональности" (extensionality): если $A \Leftrightarrow \mathrm{B} \subseteq C n(\varnothing)$, то $\mathrm{K} \div A=\mathrm{K} \div \mathrm{B}$

И в заключение может быть сформулирована важная репрезентационная теорема:

Теорема 1. (Алчуррон, Герденфорс, Макинсон)

Операция “- ” есть сокращение частичного пересечения, если и только если для него выполняются постулаты 1 - 6 .

Доказательство:

Доказательство не представляет особенных затруднений. 


\section{References}

[1] Alchourrón, C.E., Gärdenfors, P. and Makinson, D., 1985. On the logic of theory change: Partial meet contraction and revision functions. The fournal of Symbolic Logic, 50(2), pp.510-530. Available from: http://www.jstor.org/stable/2274239.

[2] Gärdenfors, P., 1988. Knowledge in flux. Modeling the dynamics of epistemic states. Cambridge, Mass., and London: The MIT Press.

[3] Rott, H., 1991. Two methods of constructing contractions and revisions of knowledge systems. Journal of Philosophical Logic, 20(2), pp.149-173. Available from: https://doi.org/ 10.1007/BF00284973. 